\title{
2-Deoxy-D-glucose (2-DG) for treatment of moderate to severely ill COVID-19 patients: A retrospective case series
}

\author{
Praveen Chandra ${ }^{*}$, Bornali Dutta ${ }^{2}$, Sandeep Mittal ${ }^{3}$, Niraj Gupta ${ }^{4}$, Sushila Kataria ${ }^{5}$, Naresh Trehan ${ }^{6}$ \\ ${ }^{1}$ Department of Structural Heart and Interventional Cardiology, Medanta The Medicity Hospital, Delhi NCR, India. \\ ${ }^{2}$ Department of Pulmonary Medicine, Medanta The Medicity Hospital, Delhi NCR, India. \\ ${ }^{3}$ Department of Respiratory and Sleep Medicine, Medanta The Medicity Hospital, Delhi NCR, India. \\ ${ }^{4}$ Department of Interventional Cardiology, Medanta The Medicity Hospital, Delhi NCR, India. \\ ${ }^{5}$ Deaprtment of Internal Medicine, Medanta The Medicity Hospital, Delhi NCR, India. \\ ${ }^{6}$ Chairman and Managing Director, Medanta The Medicity Hospital, Delhi NCR, India. \\ *Corresponding Author: Praveen Chandra, Department of Structural Heart and Interventional Cardiology, Medanta, The Medicity Hospital, \\ Delhi NCR, India.
}

Received date: 10 November 2021; Accepted date: 17 November 2021; Published date: 22 November 2021

Citation: Chandra P, Dutta B, Mittal S, Gupta N, Kataria S, et al. (2021) 2-Deoxy-D-glucose (2-DG) for treatment of moderate to severely ill COVID-19 patients: A retrospective case series. J Med Case Rep Case Series 2(15): https://doi.org/10.38207/JMCRCS/2021/0215220

Copyright: (C) 2021 Praveen Chandra. This is an open-access article distributed under the terms of the Creative Commons Attribution License, which permits unrestricted use, distribution, and reproduction in any medium, provided the original author and source are credited.

\begin{abstract}
:
Purpose: This study was conducted to assimilate evidence on the efficacy of 2-deoxy-glucose (2-DG), a glucose analog, in the treatment of moderate to severe COVID-19 patients in a hospital setting.

Patients and Methods: Medical records of adult patients with moderate to severe illness, admitted to intensive care units (ICU) of a multispecialty hospital were retrospectively analyzed. The inclusion criteria comprised of laboratory diagnosis of COVID-19 and the completeness of records. 2-DG was administered to patients on day-1 of receiving oxygen support in ICU. Details obtained from the medical record included demographics, laboratory investigations, clinical outcomes, and survival.

Results: Records of 29 patients, with an average age of $51.3 \pm 11.7$ years were included in the study. Males constituted $82.8 \%$ of the patient population. Co-morbidities (hypertension and diabetes) were present in $35.5 \%$ of patients. Standard biological markers for COVID-19 were elevated on the day of admission for all the patients. Following the 2-DG intervention, oxygen saturation levels improved to $>93 \%$ in $>70 \%$ of the patients within 7 days of intervention. The average length of the hospital stays and average time to viral clearance was 21.2 days and 11.8 days respectively. Improvement on the World Health Organization (WHO) defined ordinal scale was observed in $37.9 \%$ of the patients (movement from category 5 and 6 to category 4, i.e., from moderate/severe to mild illness) post-intervention. Mortality was observed to be $20.7 \%$ in this study. No 2-DG related side effects were observed.

Conclusion: This is the first retrospective case series examining the efficacy of 2-DG in COVID-19 management in real-world settings. In this case series, 2-DG was found to be effective in reducing oxygen requirement and time to viral clearance in moderate to severely ill COVID19 patients as reflected in improvement on the WHO ordinal scale.
\end{abstract}

Keywords: COVID-19, 2-deoxy-D-glucose, oxygen saturation, WHO ordinal scale, 2-DG

\section{Introduction}

Since the first reported case in February 2020, more than 31 million people in India have been affected by the coronavirus disease-19 (COVID-19), designated as a global pandemic by the World Health Organization(WHO).[1] This disease is caused by the highly contagious and invasive severe acute respiratory syndrome coronavirus 2 (SARS-CoV-2) and has led to more than 4 lakh deaths in the Indian subcontinent to date.[2] Concerns on the increasing rates of COVID-19 related hospitalizations, intensive care admissions, and mortality have accelerated research on deciphering the effectiveness of various therapeutic agents against this rapidly mutating virus to curb disease severity and mortality. Supportive care[3] remains the mainstay of COVID-19 management, although several repurposed and repositioned therapeutics including antivirals such as remdesivir and lopinavir alone or in combination with interferon and ribavirin are employed with varying degrees of success.[4] Other treatments such as monoclonal antibodies namely tocilizumab, and convalescent plasma therapy have shown limited success, both in trials as well as under clinical settings but are challenged by the logistics of large scale production and availability to meet the immediate needs of COVID19 patients especially at the peak of the second wave of the pandemic in developing countries. [4] 
Study on viral structure and biochemistry has shown that inhibition of glycolysis pathway is a potential target for termination of the viral replication and transmission.[5] 2-deoxy-glucose (2-DG) is an analog of glucose with an antimetabolite action.[6] It acts by inhibiting the production of glucose- 6 phosphate and instead leads to the accumulation of intracellular 2-deoxy-D-glucose-6-phosphate (2DG6P). [7] This potential therapeutic agent reduces viral load in the host cells by inhibiting glycolysis. Furthermore, in a clinical trial conducted in SARS-CoV-2 infected patients, 2-DG reduced supplemental oxygen usage and showed a higher rate of negative

\section{Material and methods}

This retrospective cohort study included 29 adult patients $(\geq 18$ years of age) admitted to the medical Intensive Care Units (ICUs) at Medanta Multispecialty Hospital, Gurugram, India during the second wave of COVID-19 between May 3 and June 28, 2021. Medical records of ICU patients with laboratory-confirmed COVID-19 infection and a need for oxygen support were included in the study. The patients received 2-DG as an adjunct to the physician-prescribed standard of care. Confirmed cases of COVID-19 were characterized by a positive result on RT-PCR assay of a throat or nasopharyngeal swab sample. Pregnant women and children $(\leq 18$ years of age) were excluded from the analysis. Ethics approval and patient consent were not required for this retrospective study as it involved only a review of medical records.

Data on patient demographics, comorbidities, clinical and laboratory findings, oxygen support requirements (room air, nasal low-flow oxygen, nasal high-flow oxygen, non-invasive ventilation (NIV) and invasive mechanical ventilation), length of hospital stay, and time to viral clearance were collected retrospectively through a review of patients' medical records during the first 14 days post-2-DG administration. Additionally, clinical improvement in patients was assessed by the patient status on WHO defined 8-point ordinal scale for clinical improvement (where a score of 1 indicates no limitation

\section{Results}

The baseline demographics and clinical characteristics of the 29 patients included in this study have been given in Table 1 . The age of patients ranged from 30-79 years with a majority $(37.9 \%)$ of the patients belonging to the age group of 41-50 years. The average age was $51.3 \pm 11.7$ years and $82.8 \%$ of the patients were male. Body mass index $(\mathrm{BMI})$ of $<25 \mathrm{~kg} / \mathrm{m}^{2}$ was noted in $72.4 \%$ of patients, with overweight and obese patients making up $17.2 \%$ and $10.3 \%$ of the patient population respectively. Co-morbidities like hypertensionand conversion of reverse transcription-polymerase chain reaction (RTPCR). Based on the promising results, the Drug Controller General of India approved the emergency use of 2-DG in moderate to severe COVID-19 patients in May 2021.[6]

There is limited clinical evidence on the use of 2-DG for the management of COVID-19 in real-world settings. The present study is the first retrospective case series to evaluate the efficacy of 2-DG and its effect on supplemental oxygen requirement in a small cohort of moderate to severely ill COVID-19 patients

in activities and 8 refers to death) [8] at the start and end of the 2-DG intervention.

Based on hospital-defined guidelines, patients admitted to ICUs were considered eligible for 2-DG intervention if they had a progressive worsening of oxygen saturation. As per hospital records, 2-DG was administered twice a day (morning and evening) for a maximum duration of ten days as an adjunct to the physician-prescribed standard of care. A pre-filled sachet of 2-DG containing $5.85 \mathrm{~g}$ of pure 2-DG consisted of a single dose. The entire contents of one sachet were dissolved in $100 \mathrm{~mL}$ potable water 1-hour before administration. The volume of the 2-DG solution was determined as per the following formula:

Volume of one dose of 2-DG $=0.77 \times$ (body weight of the patient [in $\mathrm{kgs}]) \mathrm{mL}$

Each dose of 2-DG was freshly prepared before administration and the excess 2-DG solution was discarded.

Descriptive statistics were used to summarize the data and results are reported as mean and standard deviations, as applicable. Categorical variables were summarized as counts and percentages. Missing data were not included in estimations of averages. Analysis was performed with MS Excel software (Microsoft).

diabetes mellitus were present in $24.1 \%$ and $13.8 \%$ of patients, respectively. Thirty-five percent of patients had both hypertension and diabetes. A history of smoking was present in $24.1 \%$ of patients. Elevation in key biomarkers for COVID-19 namely C-reactive proteins (CRP), D-dimer, and total leucocyte count was observed in all the patients on day-1 of 2-DG intervention. Fever, cough, and shortness of breath were the most commonly reported symptoms in all patients at admission. 
Table 1: Baseline demographic and clinical characteristics of patients treated with 2-DG

\begin{tabular}{|c|c|}
\hline Demographic Characteristics & \\
\hline Age, Mean (SD) & $51.3(11.7)$ \\
\hline Age category & n (\%) \\
\hline$<40$ years & $4(13.8)$ \\
\hline $41-50$ years & $11(37.9)$ \\
\hline $51-60$ years & $7(24.1)$ \\
\hline $61-70$ years & $5(17.2)$ \\
\hline$>70$ years & $2(6.9)$ \\
\hline Gender & n $(\%)$ \\
\hline Male & $24(82.8)$ \\
\hline Female & $5(17.2)$ \\
\hline BMI Category & $\mathrm{n}(\%)$ \\
\hline$<25$ & $21(72.4)$ \\
\hline 25.1-29.9 & $5(17.2)$ \\
\hline$>30$ & $3(10.3)$ \\
\hline Co-morbidity & n $(\%)$ \\
\hline Both hypertension and diabetes mellitus & $10(35.5)$ \\
\hline Only hypertension & $7(24.1)$ \\
\hline Only diabetes mellitus & $4(13.8)$ \\
\hline No co-morbidity & $8(27.6)$ \\
\hline Smokers, n (\%) & $7(24.1)$ \\
\hline Clinical parameters & Mean (n) \\
\hline $\mathrm{SpO}_{2}(\%)$ & $89.9(29)$ \\
\hline TLC (cells $/ \mathrm{mm}^{3}$ ) & $16.07(28)$ \\
\hline $\mathrm{CRP}(\mathrm{mg} / \mathrm{L})$ & $72.8(27)$ \\
\hline D-Dimer (ng/mL) & $1926.6(27)$ \\
\hline SGOT (units/L) & $52.04(26)$ \\
\hline SGPT (units /L) & $80.65(26)$ \\
\hline Creatinine (mg/dL) & $0.72(27)$ \\
\hline Urea (mmol/L) & $57.68(27)$ \\
\hline
\end{tabular}

Abbreviations BMI, body mass index; $\mathrm{SpO}_{2}$, oxygen saturation levels; TLC, total leucocyte count; CRP, C-reactive protein; SGOT, serum glutamic oxaloacetic transaminase; SGPT, serum glutamic-pyruvic transaminase; SD, standard deviation

Mortality of $20.7 \%$ was noted in this retrospective case series (Table 2). Six patients expired within the 14-day study period, this included 3 patients who were on invasive ventilation and 3 who were on NIV. The average length of the hospital stay was reported as 21.2 days and the time taken for the viral clearance was 11.8 days. The duration of hospital stay was between 11-20 days for 55.2\% of patients. Out of 29 patients, the RT-PCR test was repeated for 20 patients during the 14-day study period. Eighty-five percent of patients had a negative RT-PCR report at less than 15 days post-2-DG intervention (Table 2). 
Table 2 Clinical outcomes of patients treated with 2-DG

\begin{tabular}{|l|l|}
\hline Mortality, $\mathbf{n}(\boldsymbol{\%})$ & $\mathbf{6}(\mathbf{2 0 . 7})$ \\
\hline Duration of hospital stay & $\mathbf{n}(\boldsymbol{\%})$ \\
\hline $0-10$ days & $3(10.3)$ \\
\hline $11-20$ days & $16(55.2)$ \\
\hline $21-30$ days & $7(24.1)$ \\
\hline$>30$ days & $4(13.8)$ \\
\hline $\begin{array}{l}\text { Time to negative RT-PCR } \\
(\mathbf{N}=\mathbf{2 0})\end{array}$ & $\mathbf{n}(\boldsymbol{\%})$ \\
\hline$<7$ days & $8(40)$ \\
\hline$<15$ days & $17(85)$ \\
\hline$>15$ days & $3(15)$ \\
\hline
\end{tabular}

Abbreviations n/N, number; RT-PCR, reverse transcriptase-polymerase chain reaction

Improvement in oxygen saturation levels $\left(\mathrm{SpO}_{2} \%\right)$ was observed in patients treated with 2-DG. The average $\mathrm{SpO}_{2}$ levels improved from $89.9 \%$ at baseline $(n=29)$ to an average of $>95 \%$ by day 10 of 2 DG intervention in 19 patients. (Figure 1A). The decrease in the number of patients from 29 on day 1 to 19 at day 10 of follow-up is due to deaths and discharges. Eleven of the 17 patients on invasive and non-invasive high-flow oxygen at day -1 of intervention were shifted to low-flow oxygen support within 10 days of 2-DG intervention. The number of patients achieving $93 \% \mathrm{SpO}_{2}$ mark also increased with time during the study. On day 1 of intervention, 24.1 $\%$ ( 7 out of 29 patients) patients were noted to have $\mathrm{SpO}_{2}$ levels $>93$ $\%$ which increased to $70.8 \%$ (17 out of 24 patients) by day 7 and 80 $\%$ (16 out of 20 patients) by day 10 (Figure 1B). This reflects the efficacy of 2-DG in mitigating respiratory distress in COVID-19 patients in a short period.
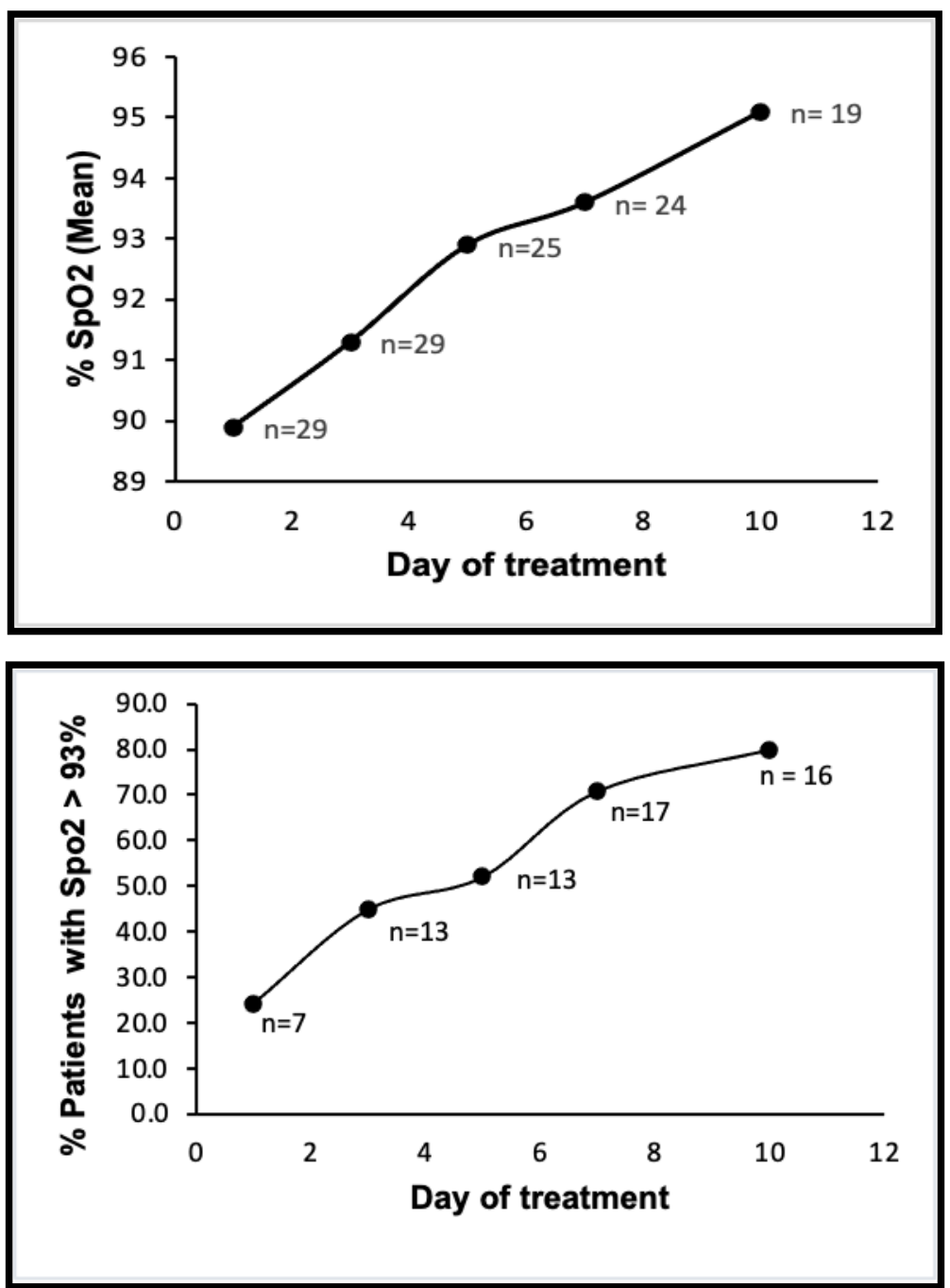

Figure 1 Improvement in oxygen saturation levels with 2-DG treatment in severely ill-COVID-19 patients.

A. Average change in $\% \mathrm{SpO}_{2}$ levels.

B. Number of patients with $\mathrm{SpO}_{2}$ levels $>93 \%$. 
The clinical condition of patients was mapped based on the 8-point WHO-defined ordinal scale ${ }^{8}$ on the day the patient received oxygen support and on the last day of the 14-day study period. During the study period, patients' WHO score ranged from 4 (hospitalized mild illness; oxygen delivered by masks or nasal prongs) to category 8 (death). Figure 2 depicts the distribution of patients based on WHO score on day 1 of 2-DG administration, considered as baseline score, and at the end of the study period. By the end of the study period, 65.5 $\%$ of the patients were in the mild disease category (ordinal score 4) as compared to $41.4 \%$ of patients at baseline (Figure 2A). Percentage of patients in ordinal score 5 (severe disease; on NIV or high-flow oxygen) reduced from a baseline of $31 \%$ to $6.9 \%$. Similarly, the percentage of patients with an ordinal score of 6 (severe illness requiring intubation or mechanical ventilation) reduced from a baseline of $27.6 \%$ to $6.9 \%$. Six patients (20.7 \%) succumbed to COVID-19 during the study period and hence fell underscore 8 of the WHO ordinal score. Graphical representation of clinical condition at baseline and end of the study for each patient has been shown in

Figure 2B. Improvement in oxygen requirement status and clinical condition was observed in 11 out of the 29 patients depicted as a decrease in ordinal score. Nine patients did not show any change in ordinal score indicating no further increase in oxygen requirement. Deterioration in clinical condition and oxygen status was observed in nine of the 29 patients over the 14- day study.
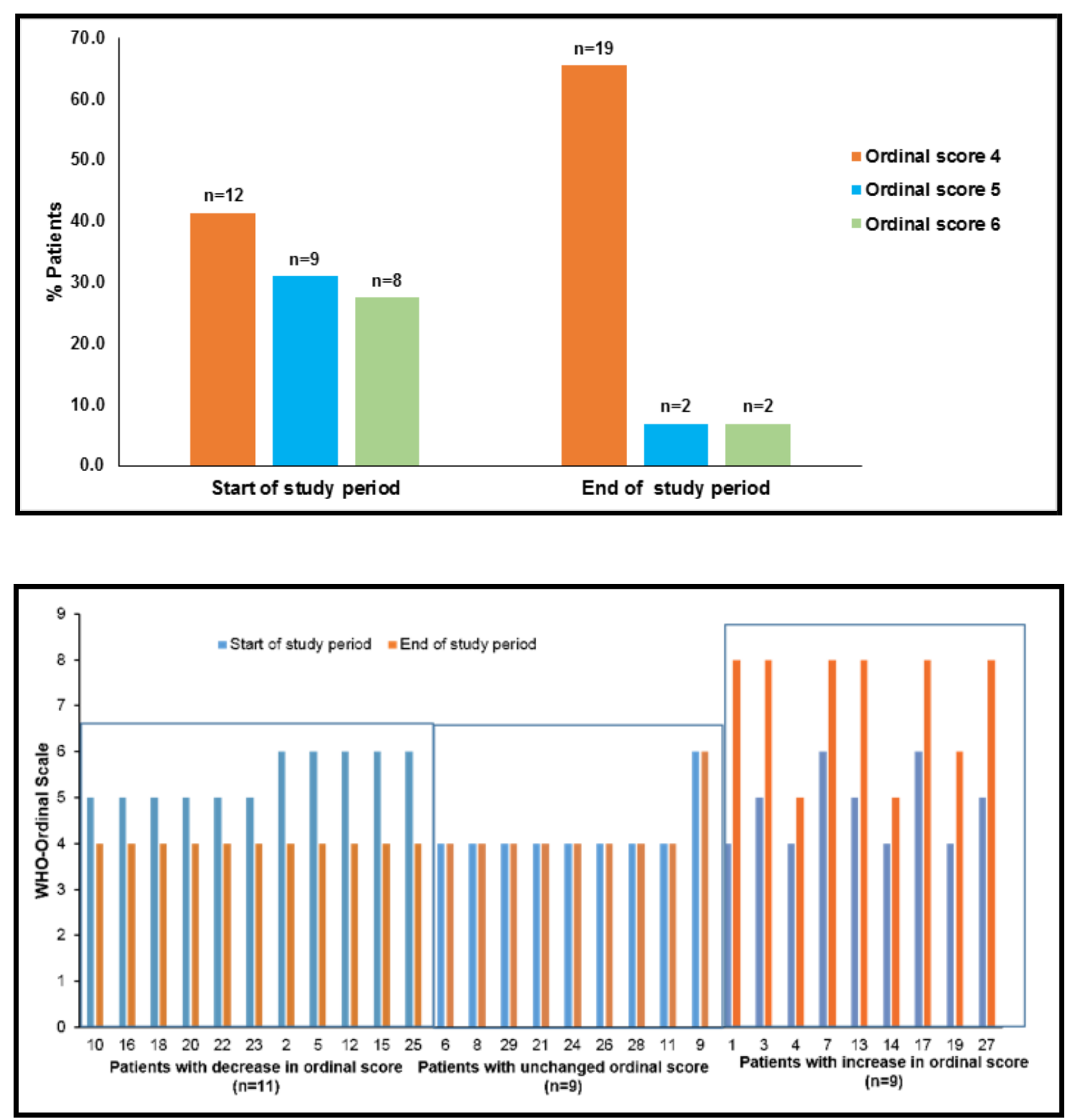

Figure 2 Clinical improvement in the patient condition represented a change in WHO defined ordinal score.

A. Patient-wise distribution based on the ordinal score at the start of the study period and the end of the study period/discharge/death.

B. Change in clinical and oxygen requirement status of individual patients based on the ordinal score over the study period. A decrease in ordinal score indicates improvement in clinical condition and vice-versa.

Overall, $37.9 \%$ of the patients $(n=11)$ showed improvement in their condition (from severely ill to mildly ill as indicated by either a 1point or 2-point improvement in ordinal score) along with a change in oxygen requirement (from mechanical ventilation and non-invasive high-flow oxygen to low-flow oxygen using masks or prongs) (Figure 3). Improvement by 2-points on the 8-point ordinal scale was observed in $17.2 \%$ of the patients $(\mathrm{n}=5)$ and improvement by 1-point was observed in $20.7 \%$ of the patients $(\mathrm{n}=6)$ (Figure 3). $31 \%$ of the patients were clinically stable indicated by no change in their WHO ordinal score status. Deterioration in medical conditions with a requirement to increased oxygen support was observed in 3 patients and 6 patients expired during the study period. 


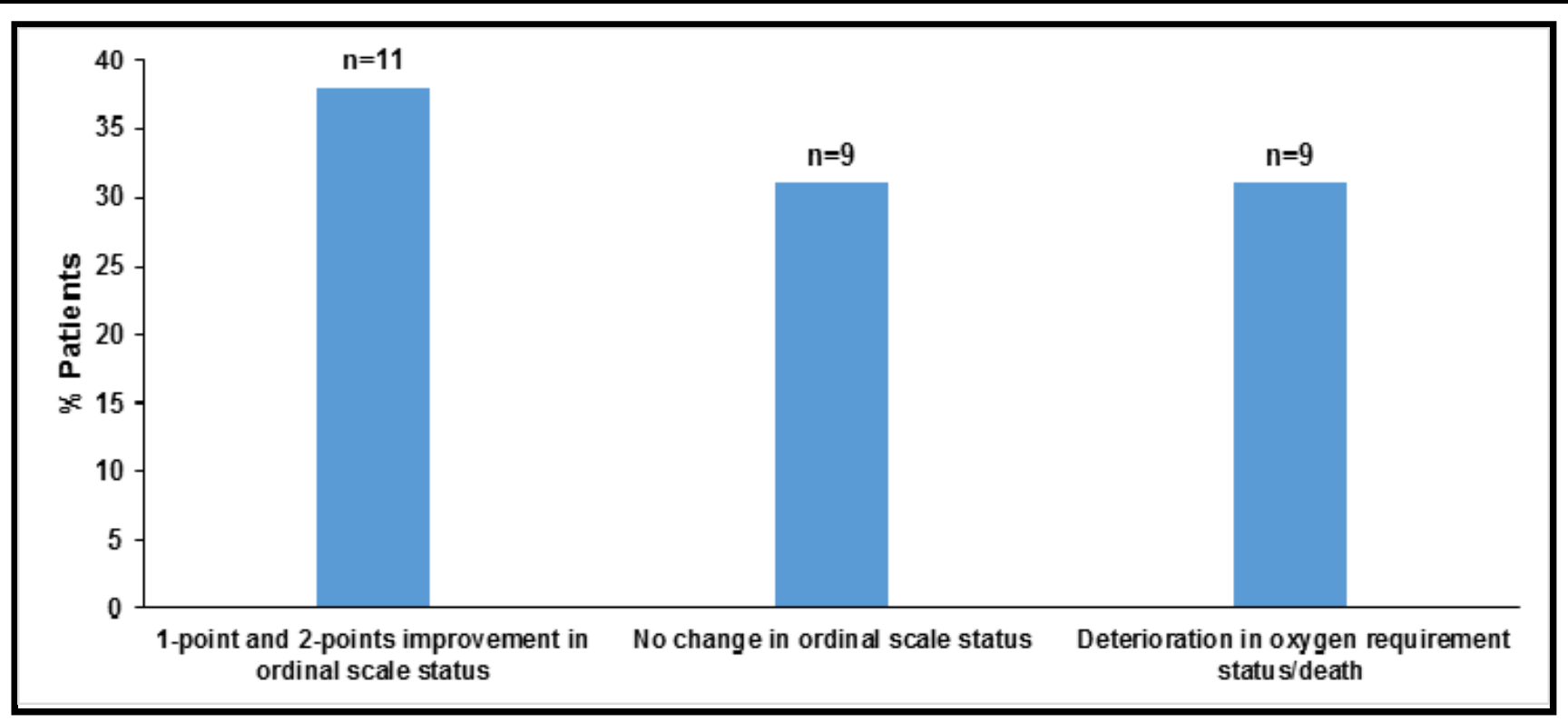

Figure 3 Distribution of patients based on the change in clinical condition and oxygen requirement status of COVID-19 patients treated with 2DG over the 14-day study period.

\section{Discussion}

This retrospective analysis of 29 patients has been done to collate evidence on the use of 2-DG as a potential therapeutic intervention in the management of COVID-19. India, like several countries, has witnessed a two-wave pattern of COVID-19 cases over the last two years. Compared to the "first wave in 2020, the 'second wave, which started in March 2021, has had a more devastating effect on the Indian population. This phase was characterized by a higher incidence of viral pneumonia with acute respiratory distress syndrome (ARDS) that resulted in significant morbidity and mortality, particularly this time involving a younger age group of patients as compared to the first wave in the preceding year.[9] It presented a significant challenge to India's health care system and overwhelmed the healthcare infrastructure with a sudden increase in the number of patients requiring supplemental oxygen. Considering this, research on the compassionate use of repurposed medication and new molecules

\section{Findings of this study shows the effect of 2-DG on the}

\section{following aspects:}

1. Oxygen requirement: As shown in Figures 1A and 1B the average $\mathrm{SpO} 2$ increased along with the number of patients at saturation of $>93 \%$ during the study period.

2. The severity of disease: As shown in Figures 2 and 3, 37.9 \% of patients showed either a 1 or 2-point improvement in WHO Ordinal Score within 15 days of 2-DG intervention.

We observed that $38 \%$ of the patients treated with 2-DG who were on high-flow oxygen or mechanical ventilation moved on to low-flow oxygen between day 10 to day 14 , with a resolution of hypoxia $\left(\mathrm{SpO}_{2}\right.$ levels $>93 \%$ ) within day 3 of intervention for $31 \%$ of the patients. Two of the six surviving patients on mechanical ventilation (33.3\%) were off intubation on day 5 , the shortest time to extubating in this study. This is comparable to previous reports on other interventions in the case of severely ill COVID-19 patients. A case report on a single remdesivir-treated severely ill COVID-19 patient showed that the patient's need for supplemental oxygen dropped over a 14-day took precedence. Consequently, in India, 2-DG, a drug originally designed for cancer treatment was studied as a treatment of COVID19 and approved by DGCI as an emergency use adjunct treatment for COVID patients.

In this study, patient outcomes in moderate to severely ill COVID-19 patients admitted to ICU and were on oxygen support have been described. Clinical responses to the intervention were observed in terms of reduction in oxygen requirement, change in oxygen requirement status, and overall improvement on the WHO- ordinal scale. 2-DG was administered between day 6 to day 7 of infection which coincides with the setting in of cytokine storm in COVID-19 patients.[10] This molecule was given as an adjunct to the standard of care. The age and gender distribution of the patient population were similar to those reported in previous studies.[11-13]

period.[14] In another large case series with 52 patients on remdesivir, improvement in the oxygen support category (from intubation to noninvasive support) was observed in $68 \%$ of the patients after a median of 18 days of treatment.[15] Studies on tocilizumab interventions have also shown a reduction in the requirement of supplemental oxygen in up to $75 \%$ of the patients with severe COVID, [16,17] with median days on mechanical ventilation of 7 days for tocilizumabtreated patients as compared to 10 days in the control cohort.[17] In this case, series, 2-DG establishes its place in the league of therapies for reducing respiratory distress in COVID-19 patients.

Around $38 \%$ of the patients showed a drop of one or two points on the WHO-ordinal scale, indicating an improvement in their health within 14 days of 2-DG administration (Figure 3). This correlates with the study by Grein et al., where $68 \%$ of patients on remdesivir reported a 2-point improvement in ordinal scale over a median of 18 days of follow-up.[15] We observed that 2-DG was beneficial to 
patients at higher levels of oxygen support such as NIV or mechanical ventilation, which is in contrast to the effect of known anti-viral remdesivir, where the benefits were higher in patients on ambient air or nasal cannula.[18] This is an important finding, considering the number of COVID-19 related deaths and morbidities seen in ICU patients in India from April to June 2021. In this case series, patients shifted from ICUs or discharged from the hospital within the study period were observed to be on low-flow oxygen support.

Another observation was that around $30 \%$ of the patients did not present any worsening of the condition during the 14-day study period. The possible role of 2-DG as a Poly pharmacological drug has been established in previous studies.[10] Anti-viral effects of 2-DG are modulated by inhibition of glycolysis[19] and production of viral glycoproteins thereby inhibiting viral multiplication as well as viral infectivity.[20] 2-DG has been also shown to exert an antiinflammatory effect. [21] The findings of this study provide further clinical evidence for use of 2-DG in COVID-19 management.

In this study, the duration of hospital stays for 19 of the 29 patients was $\leq 15$ days. Duration of hospital stay for most patients $(n=15)$ ranged between 11 to 15 days. Similar outcomes have been reported with remdesivir in a previous study, with median hospital stay between 8-11 days [22] and with tocilizumab, where mean hospital stay for severe COVID-19 patients was reported to be 28 days (RECOVERY trials). [23] Short duration to negative RT-PCR results ( $<15$ days for $85 \%$ of the patients tested during the study period) was seen in patients treated with $2-\mathrm{DG}$ as compared to the median range

\section{Conclusion}

In moderate to severe COVID-19 patients, 2-DG is effective in reducing oxygen dependence along with a shorter duration to viral clearance and a shorter duration of hospital stay. This is reflected in the overall improvement in WHO-defined ordinal scores during the 14-day study period. 2-DG has the potential to emerge as a costeffective adjunct to the standard of care. Evidence on the benefits of 2-DG to COVID-19 patients from this retrospective study can be

\section{References}

1. WHO Director-General's opening remarks at the media briefing on COVID-19 - 11 March 2020.

2. Ritchie H, Ortiz-Ospina E, Beltekian D. India: Coronavirus pandemic country profile. Our world data. 2021.

3. World Health Organization (WHO). Clinical management of COVID-19: Interim guidance. 2020.

4. Barlow A, Landolf KM, Barlow B, Yeung SYA, Heavner JJ, et al. (2020) Review of emerging pharmacotherapy for the treatment of coronavirus disease 2019. Pharmacotherapy. 40(5): 416-437.

5. Ardestani A, Azizi Z (2021) Targeting glucose metabolism for treatment of COVID-19. Signal Transduct Target Ther. 6(1): 112. of 16-38 days as reported in earlier studies on hospitalized COVID-

19 patients.[24,25] This demonstrates the efficacy of 2-DG in reducing both hospital stay duration and time to viral clearance.

The overall mortality in this case series was $20.7 \%$ with a survival rate of $79.3 \%$, which is comparable to the mortality reported in a study on tocilizumab intervention,[11] where $23 \%$ mortality was reported. In a recently published randomized, controlled trial of lopinavir-ritonavir on severe COVID-19 hospitalized patients, the 28day mortality was $22 \%$.[26] Few of the previously published case series and cohort studies majorly from China have reported mortality rates of 17 to $78 \%$ in severe COVID-19 cases, defined by the need for admission to ICU, invasive ventilation, or both. [27-30] In our study, mortality was higher in a subset of patients that had the severe disease at presentation or those who presented late (at least 10 days) after the onset of illness. This suggests that careful selection of patients with early initiation of therapy (within 10 days) can improve oxygenation thus reducing morbidity and mortality in COVID-19.

This retrospective study is limited by the small sample size, lack of a comparator group, and single-center design. Nevertheless, it provides clinical evidence that 2-DG intervention can improve outcomes in COVID-19 patients and encourages further clinical studies and randomized controlled trials. The addition of 2-DG to the standard of care is particularly vital to develop nations reeling under high healthcare costs by reducing the duration of hospital stay. No side effects or adverse events from the 2-DG intervention were observed in this case series.

further strengthened by randomized controlled trials and large-scale studies.

\section{Acknowledgments}

The authors acknowledge Molecular Connection Pvt. Ltd., Bengaluru, India received financial support from Dr. Reddy's Laboratories, India for medical writing.

Disclosure: The author reports no conflicts of interest in this work.

6. Goel R (2021) 2-Deoxy-d-glucose: from diagnostics to therapeutics. Int J Basic Clin Pharmacol. 10(6): 732.

7. Gualdoni GA, Mayer KA, Kapsch A-M, Kreuzberg K, Puck A, et al. (2018) Rhinovirus induces an anabolic reprogramming in host cell metabolism essential for viral replication. Proc Natl Acad Sci U S A. 115(30): E7158-E7165.

8. World health Organization. R\& D Blueprint: Novel Coronavirus, COVID-19 therapeutic trial synopsis. 2020.

9. Kuppalli K, Gala P, Cherabuddi K, Kalantri SP, Mohanan M, et al. (2021) India's COVID-19 crisis: a call for international action. Lancet Lond Engl. 397(10290): 2132-2135. 
10. Verma A, Adhikary A, Woloschak G, Dwarakanath BS, Papineni RVL (2020) A combinatorial approach of a polypharmacological adjuvant 2-deoxy-D-glucose with low dose radiation therapy to quell the cytokine storm in COVID-19 management. 96(11): 1323-1328.

11. Nasir N, Mahmood F, Habib K, Khanum I, Jamil B (2020) Tocilizumab for COVID-19 Acute respiratory distress syndrome: outcomes assessment using the WHO Ordinal Scale. Cureus. 12(12): e12290.

12. Colaneri M, Bogliolo L, Valsecchi P, Sacchi P, Zuccaro V, et al. (2020) Tocilizumab for treatment of severe COVID-19 patients: Preliminary Results from SMAtteo COvid19 REgistry (SMACORE). Microorganisms. 8(5): 695.

13. Kotch C, Barrett D, Teachey DT (2019) Tocilizumab for the treatment of chimeric antigen receptor $\mathrm{T}$ cell-induced cytokine release syndrome. Expert Rev Clin Immunol. 15(8): 813-822.

14. Pandya H, Jain A, Reddy P, Shah A (2020) A case report: remdesivir effective in the treatment of severe category COVID19. Int J Res Med Sci. 8(10): 3742-3745.

15. Grein J, Ohmagari N, Shin D, Diaz G, Asperges E, et al. (2020) Compassionate use of remdesivir for patients with severe Covid19. N Engl J Med. 382(24): 2327-2336.

16. Xu X, Han M, Li T, Sun W, Wang D, et al. (2020) Effective treatment of severe COVID-19 patients with tocilizumab. Proc Natl Acad Sci. 117(20): 10970-10975.

17. Kewan T, Covut F, Al-Jaghbeer MJ, Rose L, Gopalakrishna KV, et.al. (2020) Tocilizumab for treatment of patients with severe COVID-19: A retrospective cohort study. E Clinical Medicine. 24: 100418 .

18. Olalla J (2020) Remdesivir for the treatment of Covid-19 preliminary Report. N Engl J Med. 383(10): 993-994.

19. Singh D, Banerji AK, Dwarakanath BS, Tripathi RP, Gupta JP, et al. (2005) Optimizing cancer radiotherapy with 2-Deoxy-DGlucose. Strahlenther Onkol. 181(8): 507-14.

20. Passalacqua KD, Lu J, Goodfellow I, Kolawole AO, Arche JR, et al. (2019) Glycolysis is an intrinsic factor for optimal replication of a norovirus. mBio. 10(2): e02175-18.

21.
Zhao Q, Chu Z, Zhu L, Yang T, Wang P, et al. (2017) 2-Deoxy-

d-Glucose treatment decreases anti-inflammatory $\mathrm{m} 2$ macrophage polarization in mice with tumor and allergic airway inflammation. Front Immunol. 8: 637.

22. Lee S, Santarelli A, Caine K, Schritter S, Dietrich T, et al. (2020) Remdesivir for the treatment of severe COVID-19: A community hospital's experience. J Osteopath Med. 120(12): 926-933.

23. RECOVERY Collaborative Group, Horby PW, Pessoa-Amorim G, Peto L, Brightling CE, Sarkar R, et al. (2021) Tocilizumab in patients admitted to hospital with COVID-19 (RECOVERY): preliminary results of a randomised, controlled, open-label, platform trial. medRxiv. 397(10285): 1637-1645.

24. Abrahim SA, Tessema M, Defar A, Hussen A, Ejeta E, et al. (2020) Time to recovery and its predictors among adults hospitalized with COVID-19: A prospective cohort study in Ethiopia. PLOS ONE. 15(12): e0244269.

25. Audigé L, Hanson B, Kopjar B (2006) Issues in the planning and conduct of non-randomised studies. Injury. 37(4): 340-8.

26. Cao B, Wang Y, Wen D, Liu W, Wang J, et al. (2020) A trial of lopinavir-ritonavir in adults hospitalized with severe Covid-19. N Engl J Med. 382(19): 1787-1799.

27. Wang C, Wang Z, Wang G, Lau JY-N, Zhang K, et al. (2021) COVID-19 in early 2021: current status and looking forward. Signal Transduct Target Ther. 6(1): 114.

28. Wu Z, McGoogan JM (2020) Characteristics of and important lessons from the coronavirus disease 2019 (COVID-19) outbreak in China: summary of a report of 72314 cases from the Chinese Center for Disease Control and Prevention. JAMA. 323(13): $1239-1242$

29. Zhou F, Yu T, Du R, Fan G, Liu Y, et al. (2020) Clinical course and risk factors for mortality of adult inpatients with COVID-19 in Wuhan, China: a retrospective cohort study. Lancet Lond Engl. 395(10229): 1054-1062.

30. Bhatraju PK, Ghassemieh BJ, Nichols M, Kim R, Jerome KR, et al. (2020) Covid-19 in critically ill patients in the Seattle region case Series. N Engl J Med. 382(21): 2012-22. 